
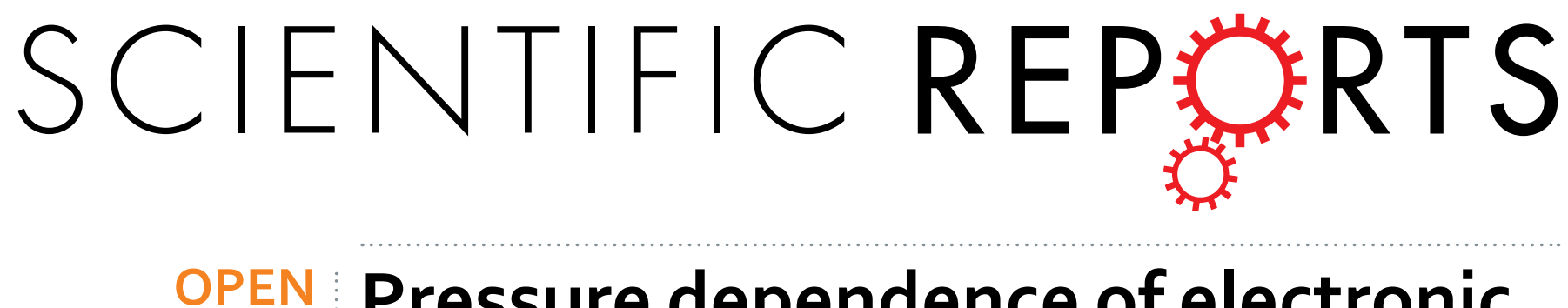

\title{
Pressure dependence of electronic structure and superconductivity of the $\operatorname{MnX}(X=N, P, A s, S b)$
}

Received: 07 October 2015

Accepted: 01 February 2016

Published: 23 February 2016
XiaoYu Chong ${ }^{1}$, YeHua Jiang ${ }^{1}$, Rong Zhou ${ }^{1}$ \& Jing Feng ${ }^{1,2}$

A recently experimental discovered (Cheng et al., Phys. Rev. Lett. 114, 117001 (2015)) of superconductivity on the border of long-range magnetic order in the itinerant-electron helimagnet $\mathrm{MnP}$ via the application of high pressure makes $\mathrm{MnP}$ the first $\mathrm{Mn}$-based superconductor. In this paper, we carry out first-principles calculations on $\mathrm{MnX}(X=N, P, A s, S b)$ and find superconducting critical temperature $T_{\mathrm{C}}$ of $\mathrm{MnP}$ sharply increases near the critical pressure $\mathrm{P}_{\mathrm{C}} \approx 8 \mathrm{GPa}$, which is in good agreement with the experiments. Electron-phonon coupling constant $\lambda$ and electronic density of states at the Fermi level $N\left(E_{\mathrm{F}}\right)$ are found to increase with pressure for MnP, which lead to the increase of $T_{C}$ of MnP. Moreover, we also find that the $T_{C}$ of MnAs and MnSb are higher than MnP, implying that the MnAs and MnSb may be the more potential Mn-based superconducting materials.

Superconductivity has been deeply studied and developed very quickly since its discovery in 1911. But many difficult problems about it have not been solved. For example, the most distinguished problem of unconventional superconductivity (SC) as found in several distinct superconducting systems including the heavy-fermion, organic, cuprates, and the iron-based superconductors can be generally described in the framework of the antiferromagnetic quantum critical point $(\mathrm{QCP})^{1-4}$. The critical spin fluctuations would play a crucial role for mediating the Cooper pairs ${ }^{3}$. Moreover, In order to realize a magnetic QCP, an effective approach should be provided to search new classes of unconventional superconductors. The discovery of Cr-based unconventional superconductor has left manganese (Mn) the only $3 \mathrm{~d}$ element that does not show SC among any Mn-based compounds ${ }^{5}$. The itinerant-electron helimagnet $\mathrm{MnP}^{6}$ has a much reduced moment of $\sim 1.3 \mu \mathrm{B} / \mathrm{Mn}$ and the strong magnetism of $\mathrm{Mn}$ is commonly believed to be antagonistic to SC. Nevertheless, J.-G. Cheng and K. Matsubayashi discover the superconductivity on the border of long-range magnetic order in the itinerant-electron helimagnet MnP under high pressure in experiment recently ${ }^{7}$. The synthesized needle-shaped MnP single crystals have an orthorhombic B31-type structure with lattice constants $a=5.26, b=3.17$, and $c=5.92 \AA$, respectively. They found that superconductivity with $\mathrm{T}_{\mathrm{SC}} \approx 1 \mathrm{~K}$ emerges and exists merely near the critical pressure $\mathrm{P}_{\mathrm{C}} \approx 8 \mathrm{GPa}$, which can be attributed to the external pressure inhibiting the antiferromagnetic order and inducing superconductivity. So far, there are no theoretical results to verify the superconducting transition. In this paper, we present a systematic investigation of the high pressure behaviors of $\mathrm{MnX}(\mathrm{X}=\mathrm{N}, \mathrm{P}, \mathrm{As}, \mathrm{Sb})$, including the electronic sructures, elastic properties and mechanisms of superconductivity. The main purpose of this paper is only to supply a new idea and perspective to understand the mechanism of superconducting transition of MnP under high pressure.

\section{Results and Discussion}

Structure and chemical bonding. The crystal structure of $\mathrm{MnX}(\mathrm{X}=\mathrm{N}, \mathrm{P}, \mathrm{As}, \mathrm{Sb})$ with an orthorhombic B31-type structure are shown in Fig. 1 . The $\mathrm{Mn}$ and $\mathrm{X}$ ions form an unique edge-sharing $\mathrm{MnX}_{6}$ octahedron in the lattice. The crystal also can be regarded as the $\mathrm{Mn}$ and $\mathrm{P}$ alternate layers structure. Furthermore, the chemical bond lengths change of $\mathrm{MnP}$ with the pressure increasing is shown in Fig. 2. The P-Mn and P-P bond length decrease with the external pressure increasing. But it is strange that the length of two kinds of P-Mn bonds sharply increase when the external pressure up to $8.13 \mathrm{GPa}$ compared with $6.17 \mathrm{GPa}$.

Electronic structure. We calculate the spin partial density of states for the $\mathrm{MnN}, \mathrm{MnP}, \mathrm{MnAs}$ and $\mathrm{MnSb}$ at 0.0001 GPa, 1.98 GPa, 4.02 GPa, 6.17 GPa, 8.13 GPa and 9.69 GPa and the results of $\mathrm{MnP}$ are shown in Fig. 3. It can

${ }^{1}$ Faculty of Material Science and Engineering, Kunming University of Science and Technology, Kunming 650093, People's Republic of China. 'School of Engineering and Applied Sciences, Harvard University, Cambridge, MA 02138, USA. Correspondence and requests for materials should be addressed toY.J. (email: jiangyehua@kmust.edu.cn) and J.F. (email: jfeng@seas.harvard.edu) 
(b)

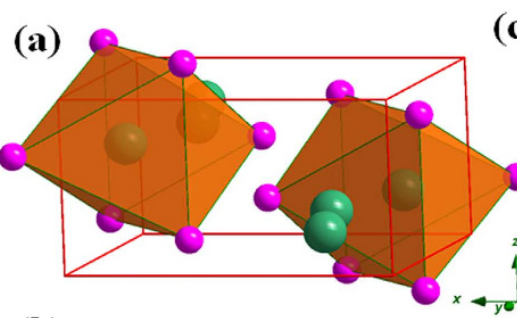

(c)

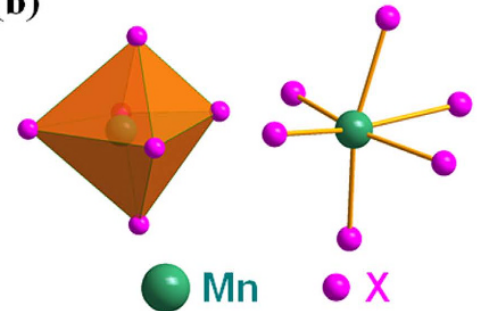

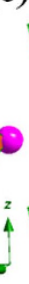

(c)

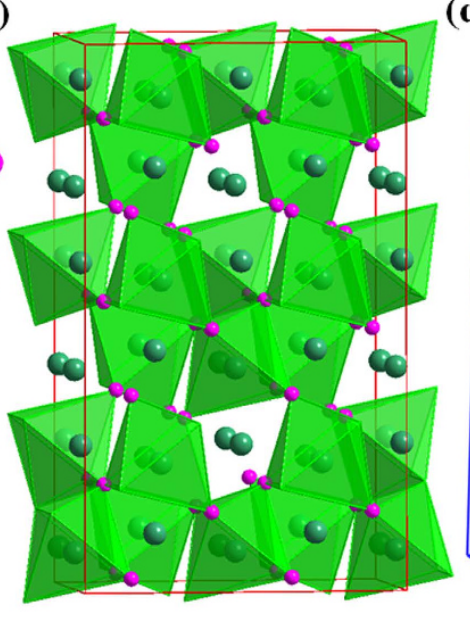

(d)

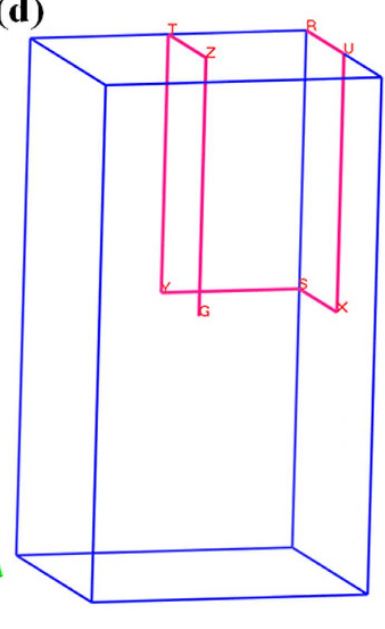

Figure 1. Crystal structure of $\operatorname{MnX}(X=N, P, A s, S b)$. (a) The unit cell of $\mathrm{MnX}$; (b) The coordination polyhedrons for the $\mathrm{Mn}$ atoms, which is a $\mathrm{MnX}_{6}$ octahedron structure; (c) The $3 \times 2 \times 2$ supercell of $\mathrm{MnX}$; (d) Schematic representation of the high-symmetry points in the first Brillouin zone for orthorhombic system $\mathrm{MnX}: \mathrm{G}(0,0,0) \rightarrow \mathrm{Z}(0,0,0.5) \rightarrow \mathrm{T}(-0.5,0,0.5) \rightarrow \mathrm{Y}(-0.5,0,0) \rightarrow \mathrm{S}(-0.5,0.5,0) \rightarrow \mathrm{X}(0,0.5,0) \rightarrow \mathrm{U}(0,0.5$, $0.5) \rightarrow \mathrm{R}(-0.5,0.5,0.5)$.

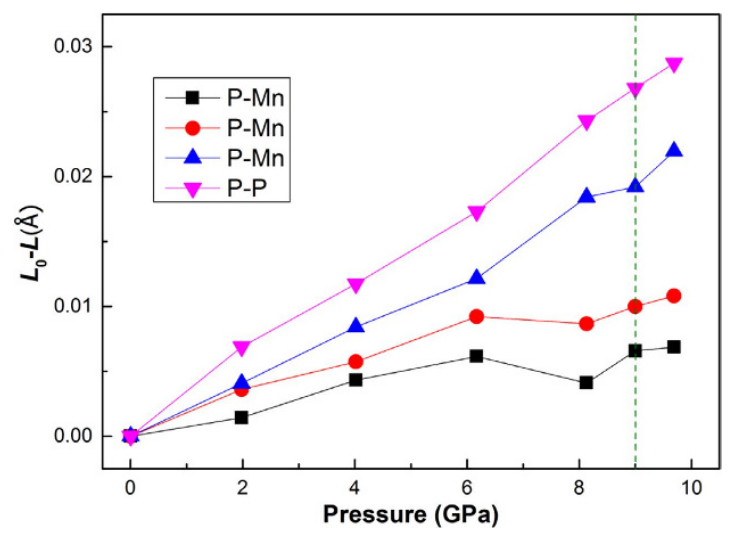

Figure 2. The bond lengths change of MnP wtith the pressure increasing. $\mathrm{L}_{0}$ is the bond length under $0.0001 \mathrm{GPa}$ and $\mathrm{L}$ is the bond length under higher pressure.

be seen that the $\mathrm{MnP}$ at different pressure up to $10 \mathrm{GPa}$ all have metallic characteristics because the DOS at Fermi level are not zero, which might favor the superconducting behavior. Mn atoms contribute more to DOS than the $\mathrm{P}$ atoms at Fermi level and the majority of the density of states near the Fermi level for MnP is attributed to the $\mathrm{Mn}-3 \mathrm{~d}$ states. The P-3p bands are overlapped with the Mn-3d bands in the $-10-8 \mathrm{eV}$ energy range, representing a hybridization of the P-3p and Mn-3d states to form the covalent bonding. The difference of the spin-up band and spin-down band of Mn-3d orbitals show that they carry very large spin moment in $\mathrm{MnP}$ at different pressure. $\mathrm{P}-3 \mathrm{p}$ and $\mathrm{P}-3 \mathrm{~s}$ orbitals also have small contribution to the magnetic property of MnP. Literature ${ }^{6}$ reveal that $\mathrm{MnP}$ undergoes two successive magnetic transitions upon cooling in the absence of a magnetic field. One is a transition from the paramagnetic $(\mathrm{PM})$ to ferromagnetic $(\mathrm{FM})$ state at $T_{\mathrm{C}}=291 \mathrm{~K}$, and then a second transition to a double helical state at $T_{\mathrm{s}} \approx 50 \mathrm{~K}$, In the FM state, the ordered moment of the Mn spins is about $1.3 \mu_{B} / \mathrm{Mn}$. Moreover, as shown in Fig. 3, the pressure up to $10 \mathrm{GPa}$ have less effect on the density of states of MnP. Furthermore, the total density of states at Fermi surface $\left(N\left(E_{F}\right)\right)$ of $\mathrm{MnP}$ are summarized in Table S1 and increase with the pressure increasing.

Vibrational analysis. The calculated phonon dispersions and projected phonon densities of states (PHDOS) of $\mathrm{MnP}$ under different pressure are shown in Fig. 4. Absence of any imaginary frequency in the Brillouin zone confrms the dynamical stability of MnP. The modes at the high frequency region are associated with the vibrations of $\mathrm{P}$ atoms beating against $\mathrm{Mn}$ atoms. The PHDOS of this structure shows that the heavier Mn atoms dominate the low-frequency vibrations, and the lighter $\mathrm{P}$ atoms contribute significantly to the high-frequency modes. The phonon calculation results for other Mn-based compounds at different pressure can be seen in Figure S1. 

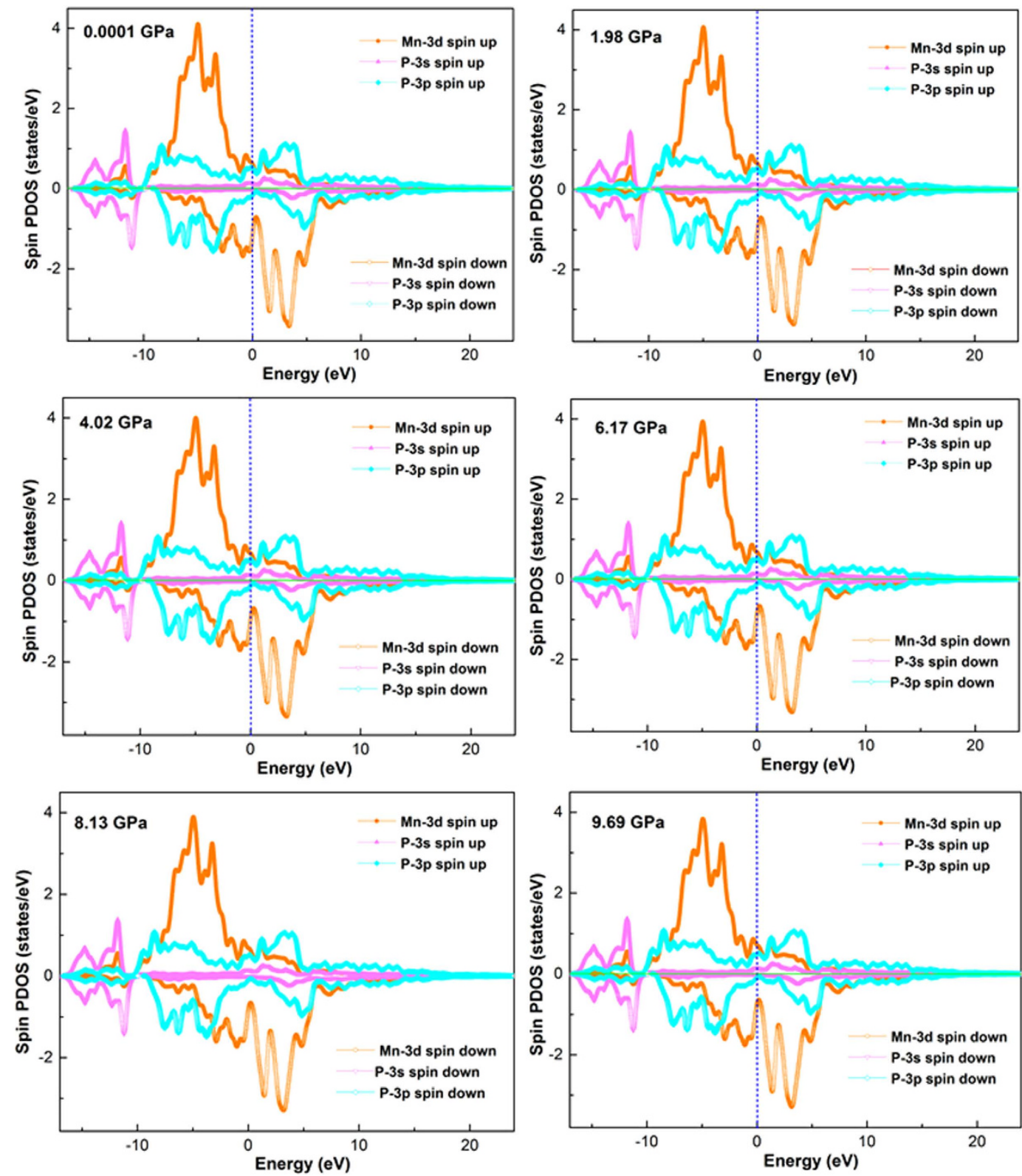

Figure 3. The calculated spin polarized partial density of states (SPDOS) of MnP at different pressure. The blue dash vertical line represents the Fermi energy. The unit of SPDOS is states/eV/unit cell.

Superconductivity properties. The superconductivity of the selected structures can be conveniently studied by electron-phonon coupling (EPC) calculation. The superconducting critical temperature can be estimated from the McMillan formula ${ }^{8,9}$ given in equation (1)

$$
T_{C}=\frac{\Theta_{D}}{1.45} \exp \left\{\frac{-1.04(1+\lambda)}{\lambda-\mu^{*}(1+0.62 \lambda)}\right\}
$$

where $\Theta_{\mathrm{D}}$ is the Debye temperature, $\lambda$ is the electron-phonon coupling strength, $\mu^{*}$ is the Coulomb pseudopotential. MaMillan's strong coupling theory defines an electron-phonon coupling constant (EPC) $\lambda$ by ${ }^{8,10,11}$

$$
\lambda=\frac{\eta}{M\left\langle\omega^{2}\right\rangle^{\frac{1}{2}}}=\frac{N\left(E_{F}\right)\left\langle I^{2}\right\rangle}{M\left\langle\omega^{2}\right\rangle^{\frac{1}{2}}}
$$

where $M$ is the atomic mass, $\left\langle I^{2}\right\rangle$ is the square of the electron-ion matrix element, $\left\langle\omega^{2}\right\rangle^{\frac{1}{2}}$ is the average squared phonon frequency, $N\left(E_{F}\right)$ is the total density of states at Fermi surface which can be found in Table S1. Furthermore, $\mu^{*}$ can be obtained from the empirical relation in the following equation: 

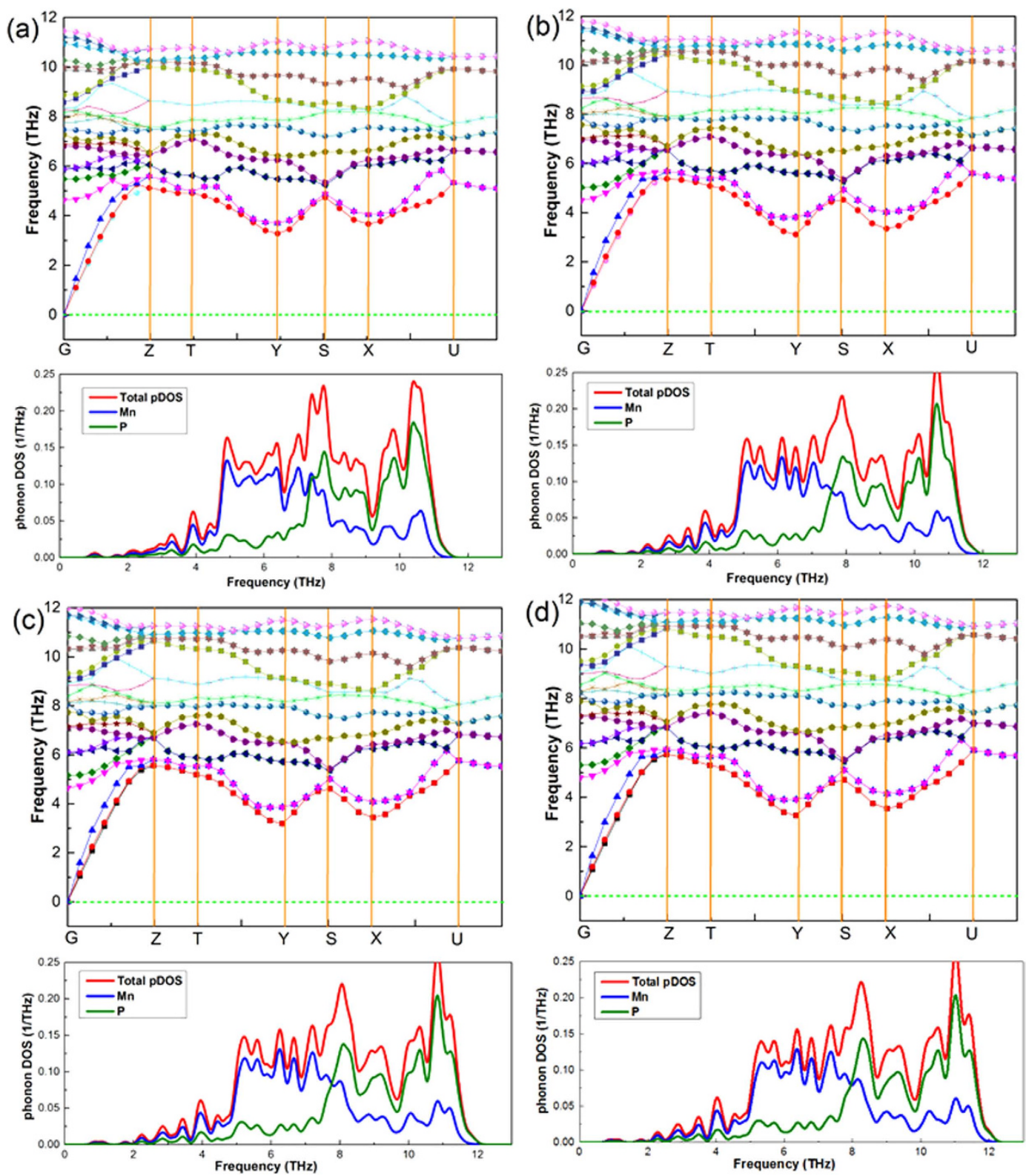

Figure 4. The phonon dispersions and projected phonon densities of states (PHDOS) of MnP under different pressure. (a) $0.0001 \mathrm{GPa}$; (b) $4.02 \mathrm{GPa}$; (c) $6.17 \mathrm{GPa}$; (d) $8.13 \mathrm{GPa}$.

$$
\mu^{*}=\frac{0.26 N\left(E_{F}\right)}{1+N\left(E_{F}\right)}
$$

In this paper, $\Theta_{\mathrm{D}}$ is calculated using the following expression ${ }^{12}$ :

$$
\Theta_{D}=\frac{h}{k_{B}}\left[\frac{3 n}{4 \pi}\left(\frac{N_{A} \rho}{M}\right)\right]^{1 / 3} v_{m}
$$

where $h$ and $k$ are the Planck and Boltzmann constants, respectively. $N_{\mathrm{A}}$ is Avogadro's number, $n$ is the number of atoms in the molecule, $M$ is the molecular weight, and $\rho$ is the density of the crystal. $v_{\mathrm{m}}$ is the mean sound velocity, which can be calculated by ${ }^{13}$

$$
v_{m}=\left[\frac{1}{3}\left(\frac{2}{v_{t}^{3}}+\frac{1}{v_{l}^{3}}\right)\right]^{-1 / 3}
$$

In the above equation, $v_{\mathrm{t}}$ and $v_{1}$ are the transverse and longitudinal sound velocities obtained by ${ }^{14}$ 

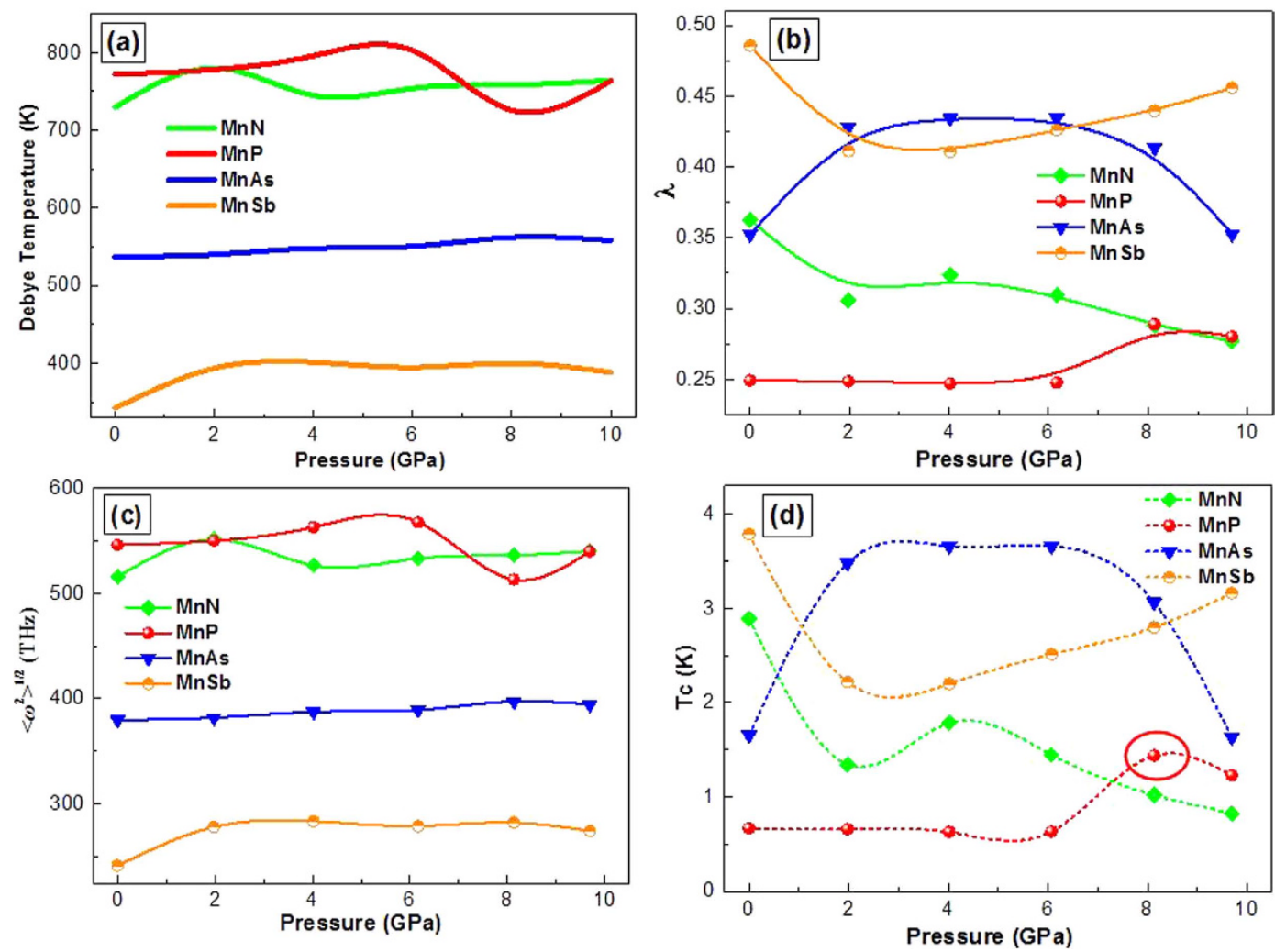

Figure 5. The calculated Debye temperature $\Theta_{\mathrm{D}}(\mathbf{a})$, electron-phonon coupling strength $\lambda(\mathbf{b})$, average phonon frequency $\left.<\omega^{2}\right\rangle^{1 / 2}(\mathbf{c})$ and superconducting critical temperature $T_{\mathrm{C}}(\mathbf{d})$ of $\mathrm{MnX}(\mathrm{X}=\mathrm{N}, \mathrm{P}, \mathrm{As}, \mathrm{Sb})$ as a function of pressure.

$$
\begin{gathered}
v_{l}=\sqrt{\frac{(B+(4 / 3) G)}{\rho}} \\
v_{t}=\sqrt{\frac{G}{\rho}}
\end{gathered}
$$

where $B$ and $G$ are the bulk modulus and shear modulus, respectively. In order to calculate the $B$ and $G$, firstly, the elastic constants of orthorhombic crystal $\left(C_{11}, C_{22}, C_{33}, C_{44}, C_{55}, C_{66}, C_{12}, C_{13}\right.$ and $\left.C_{23}\right)$ are calculated by applying stress tensors with various small strains onto the equilibrium structures. After obtaining elastic constants, the polycrystalline bulk modulus $B$ and shear modulus $G$ are calculated from the Voigt-Reuss-Hill (VRH) approximations ${ }^{15}$. The calculated density and mechanical modulus are tabulated in Table S2. The evaluated Debye temperature $\Theta_{\mathrm{D}}$, electron-phonon coupling strength $\lambda$, average phonon frequency $\left\langle\omega^{2}\right\rangle^{1 / 2}$ and superconducting critical temperature $T_{\mathrm{C}}$ are exhibited in Fig. 5. The variation trend of Debye temperature $\Theta_{\mathrm{D}}$ and average phonon frequency $\left\langle\omega^{2}\right\rangle^{1 / 2}$ are similar and the $\Theta_{\mathrm{D}}$ and $\left\langle\omega^{2}\right\rangle^{1 / 2}$ values of $\mathrm{MnP}$ and $\mathrm{MnN}$ are larger than MnAs and MnSb. Moreover, the electron-phonon coupling strength $\lambda$ and superconducting critical temperature $T_{\mathrm{C}}$ has the similar variation trend and the $\lambda$ and $T_{\mathrm{C}}$ values of $\mathrm{MnP}$ and $\mathrm{MnN}$ are smaller than MnAs and MnSb, suggesting that the MnAs and MnSb may be the more potential Mn-based superconducting materials than MnP and MnN. The EPC parameter $\lambda$ of the compounds is below 0.5 , which indicate the electron-phonon interaction is fairly weak. Although the $\Theta_{\mathrm{D}}$ of $\mathrm{MnAs}$ and $\mathrm{MnSb}$ are lower than $\mathrm{MnP}$ and $\mathrm{MnN}$, the larger EPC parameter $\lambda$ can mainly directly contribute to higher $T_{C}$ of MnAs and MnSb. In consideration of the $N\left(E_{f}\right)$ values in Table S1, we can infer that the weak electron phonon coupling $\lambda$ and small $N\left(E_{f}\right)$ are the main factors, which lead to the low $T_{C}$ of $\mathrm{MnP}$ and $\mathrm{MnN}^{16}$. As has been reported, the application of high pressure reduces continuously the magnetic transition temperatures and eventually suppresses the magnetic order around $P_{C} \approx 8 \mathrm{GPa}^{7}$. With the pressure increasing, the decrease of $\left\langle\omega^{2}\right\rangle^{1 / 2}$ play an important role to the upward trend of the EPC parameter $\lambda$ of MnP when the pressure is near $8 \mathrm{GPa}$. Meanwhile $N(F)$ of $\mathrm{MnP}$ also increases under the studied pressure range. The tendency of the two parameters makes $\lambda$ become higher, which lead to the increase of $T_{C}$ of $\mathrm{MnP}$ with increasing pressure. Our computational results are in accordance with the experimental observation in the framework of BCS superconductivity and the deep reason need to be further investigated. 


\section{Conclusion}

In summary, the electronic structure, lattice dynamics, elastic properties and superconductivity of $\mathrm{MnX}(\mathrm{X}=\mathrm{N}$, $\mathrm{P}, \mathrm{As}, \mathrm{Sb})$ are investigated by means of the first-principles within the LSDA+U method. The majority of the density of states near the Fermi level for $\mathrm{MnP}$ is attributed to the $\mathrm{Mn}-3 \mathrm{~d}$ states and the total density of states at Fermi surface $\left(N\left(E_{F}\right)\right)$ of MnP increase with the pressure increasing. The increasing EPC parameter $\lambda$ makes the superconducting critical temperature $T_{\mathrm{C}}$ of itinerant helimagnet $\mathrm{MnP}$ become higher than $1 \mathrm{~K}$ when its long-range magnetic order is completely suppressed by the application of high pressure around $P_{C} \approx 8 \mathrm{GPa}$, which is in consistent with the experimental observation and provide theoretical identification for the experimental finding that breaks the general wisdom about the Mn's antagonism to superconductivity. In addition, the $T_{C}$ of MnAs and $\mathrm{MnSb}$ are found to be higher than MnP, which indicates that the MnAs and MnSb may be the more potential Mn-based superconducting materials. This work would provide guidelines for future experimental investigations and hope that such an investigation might contribute some further understanding to the superconductivity of $\mathrm{MnP}$ under high pressure.

\section{Methods}

In this paper, the electronic structure calculations with high accuracy for the stable $\mathrm{MnX}(\mathrm{X}=\mathrm{N}, \mathrm{P}, \mathrm{As}, \mathrm{Sb})$ are performed using the on-the-fly generated (OTFG) pseudopotentials ${ }^{17}$ implemented in Cambridge Serial Total Energy Package (CASTEP) code based on the density functional theory (DFT). The exchange-correlation energy is calculated using local spin-polarized density approximation (LSDA). For strong correlated systems, these functionals are unable to give the correct ground state. we have selected the LSDA $+U$ ( $U$ is the Hubbard energy) method in this calculation. The $U$ values are tested and selected by experiment and theory from the references. The $\mathrm{U}$ value is chosen as $6 \mathrm{eV}$ in this wok. The dispersion interactions correction proposed by Grimme is considered in terms of DFT + D2 scheme in this work ${ }^{18}$. For different atomic species, the valence orbitals and electrons for pseudo-atoms are Mn $3 d^{5} 4 s^{2}, N 2 s^{2} 2 p^{3}, P 3 s^{2} 3 p^{3}$, As $4 s^{2} 4 p^{3}$ and $S b 5 s^{2} 5 p^{3}$. The electronic wave functions are expanded in a plane-wave basis set with a cutoff energy of $800 \mathrm{eV}$ and appropriate Monkhorst-Pack mesh of $4 \times 6 \times 8$ is chosen for all compounds to ensure that enthalpy calculations are well converged to better than $1 \mathrm{meV} /$ atom. In the geometrical optimization, all forces on atoms are converged to less than $0.005 \mathrm{eV} / \AA$. The phonon calculations and electron-phonon coupling (EPC) calculations are carried out using the linear response theory through the Quantum ESPRESSO package ${ }^{19}$. The kinetic energy cutoff is set $90 \mathrm{Ry}$. And the $q$-point mesh of the electron-phonon interaction matrix element adopted $4 \times 4 \times 4$.

\section{References}

1. Mathur, N. D. et al. Magnetically mediated superconductivity in heavy fermion compounds. Nature 394, 39 (1998).

2. Norman, M. R. The challenge of unconventional superconductivity. Science 332, 196 (2011).

3. Monthoux, P., Pines, D. \& Lonzarich, G. G. Superconductivity without phonons. Nature 450, 1177 (2007).

4. Gegenwart, P., Si, Q. \& Steglich, F. Quantum criticality in heavy-fermion metals. Nat. Phys. 4, 186 (2008).

5. Cheng, W. W. J.-G. et al. Broad temperature plateau for thermoelectric figure of merit ZT $>2$ in phase-separated PbTe0.7S0.3. Nat. Commun. 5, 5508 (2014).

6. Huber, E. E. J. \& Ridgley, H. D. Magnetic properties of a single crystal of manganese phosphide. Phys. Rev. 135, A1033 (1964).

7. Cheng, J. G. et al. Pressure induced superconductivity on the border of magnetic order in MnP. Phys. Rev. Lett. 114, 117001 (2015).

8. McMillan, W. L. Transition temperature of strong-coupled superconductors. Phys. Rev. B 167, 331 (1968).

9. Xu, Y., Chen, C. \& Wu, B. Superconductivity in ordered LiBe alloy under high pressure: A first-principles study. Solid State Commun. 152, $151(2012)$

10. Ashcroft, N. W. Hydrogen dominant metallic alloys: high temperature superconductors? Phys. Rev. Lett. 92, 187002 (2004).

11. Zhang, H. et al. High-temperature Superconductivity in compressed Solid Silane. Sci. Rep. 5, 8845 (2015).

12. Chong, X. Y., Jiang, Y. H., Zhou, R. \& Feng, J. Electronic structures mechanical and thermal properties of V-C binary compounds. RSC Adv. 4, 44959 (2014)

13. Feng, J., Xiao, B., Zhou, R., Pan, W. \& Clarke, D. R. Anisotropic elastic and thermal properties of the double perovskite slab-rock salt layer $\mathrm{Ln}_{2} \mathrm{SrAl}_{2} \mathrm{O}_{7}(\mathrm{Ln}=\mathrm{La}, \mathrm{Nd}, \mathrm{Sm}, \mathrm{Eu}, \mathrm{Gd}$ or Dy) natural superlattice structure. Acta Mater. 60, 3380 (2012).

14. Feng, J. et al. Calculation of the thermal conductivity of $\mathrm{L}_{2} \mathrm{SrAl}_{2} \mathrm{O}_{7}(\mathrm{~L}=\mathrm{La}, \mathrm{Nd}, \mathrm{Sm}, \mathrm{Eu}, \mathrm{Gd}, \mathrm{Dy})$. Phys. Rev. B 84, 024302 (2011).

15. Hill, R. The elastic behaviour of a crystalline aggregate. Phys. Phys. Soc. Sec. A 65, 349 (1952).

16. Liu,Y. et al. First-principles study on the structural and electronic properties of metallic $\mathrm{HfH}_{2}$ under pressure. Sci. Rep. 5, 11381 (2015).

17. Vanderbilt, D. Soft self-consistent pseudopotentials in a generalized eigenvalue formalism. Phys. Rev. B 41, 7892 (1990).

18. Grimme, S. Semiempirical GGA-type density functional constructed with a long-range dispersion correction. J. Comput. Chem. 27, 1787 (2006).

19. Giannozzi, P., Baroni, S., Bonini, N. \& Calandra, M. QUANTUM ESPRESSO: a modular and open-source software project for quantum simulations of materials. J. Phys.: Condens. Matter 21, 395502 (2009).

\section{Acknowledgements}

This work was supported by the Academic Newcomer Award of Doctoral Students in Yunnan Province (2014).

\section{Author Contributions}

X.C. performed the calculation and wrote the main part of the paper. Y.J. and R.Z. designed the research and analyzed the data. J.F. provided the idea and participated in the discussion.

\section{Additional Information}

Supplementary information accompanies this paper at http://www.nature.com/srep

Competing financial interests: The authors declare no competing financial interests.

How to cite this article: Chong, X. Y. et al. Pressure dependence of electronic structure and superconductivity of the MnX (X =N, P, As, Sb). Sci. Rep. 6, 21821; doi: 10.1038/srep21821 (2016). 
(c) (i) This work is licensed under a Creative Commons Attribution 4.0 International License. The images or other third party material in this article are included in the article's Creative Commons license, unless indicated otherwise in the credit line; if the material is not included under the Creative Commons license, users will need to obtain permission from the license holder to reproduce the material. To view a copy of this license, visit http://creativecommons.org/licenses/by/4.0/ 